\title{
Applying Correlative Brightfield, Fluorescence, and Raman Microscopy to Determine and Track Component Locations in Formulations
}

\author{
D.J. Palatini, S.L. Zhang, B. Aral, and H. Crookham
}

Unilever Research U.S., Edgewater Laboratory, 45 River Road, Edgewater, New Jersey 07020

Heterogeneous mixtures of ingredients that result in a solid or liquid product can combine and/or migrate in various manners during processing. The important properties (activity, stability, etc.) that define the qualities of these products are strongly affected by the microstructure of the resultant combination. It is therefore critical that methods be available to rapidly and accurately determine the location of components in products to provide a clearer understanding of the effects of processing changes and ingredient modifications. Knowledge in this area can provide valuable information in the design and manufacture of new and improved products.

Conventional brightfield light microscopy is a well-established technique that can provide valuable spatial information regarding the microstructure of a formulation, however, it cannot provide knowledge regarding the location of the components unless some type of staining is applied. Fluorescence microscopy can be successfully applied to locate components in a mixture when the component(s) of interest have an affinity for a specific fluorophore. At times, however, a question may exist regarding the faithfulness of the probe to "tag" only the desired component after a product is manufactured via a complex process. Raman microscopy uses the inherent spectral signatures of molecules and does not rely on staining or tagging to chemically map the microstructure. Confocal Raman microscopy can selectively sample any given xyz location in a sample with micrometer spatial resolution. Two-dimensional chemical maps are generated by employing an automated xy stage and 3 dimensional images are produced by sequentially acquiring a set of xy slices at different $\mathrm{z}$ depths. Therefore, Raman microscopy can be used as a test of the accuracy of fluorescence tagging. It is also possible to combine the results of these three types of microscopy to provide additional information about the location of specific components of interest in a formulation.

Figures $1 \mathrm{~A}$ and $2 \mathrm{~A}$ are brightfield light photomicrographs of regions of a solid formulation that has been applied onto a microscope slide. Figures $1 \mathrm{~B}$ and $2 \mathrm{~B}$ are reflected light fluorescence photomicrographs of the same area. A fluorochrome that exhibits affinity to a specific component of interest in the formulation was added during processing and can be visualized here. This method of determining the location of this component is time efficient and is the desired tracking method. However, a technique was desired to confirm the accuracy of the tagging. Figures $1 \mathrm{C}$ and $2 \mathrm{C}$ are the resultant Raman chemical maps of the component of interest (as seen via fluorescence tagging in Figures $1 \mathrm{~B}$ and $2 \mathrm{~B}$ ). The Raman maps show excellent correlation not only in location but also in intensity of the fluorescence signal that can be related to the amount of the actual material at each specific region. With this agreement established, the more time efficient fluorescence tagging method can be utilized to track these components.

These examples provide a demonstration of the application of three microscopy techniques to determine the location of components in a complex, heterogeneous mixture. 

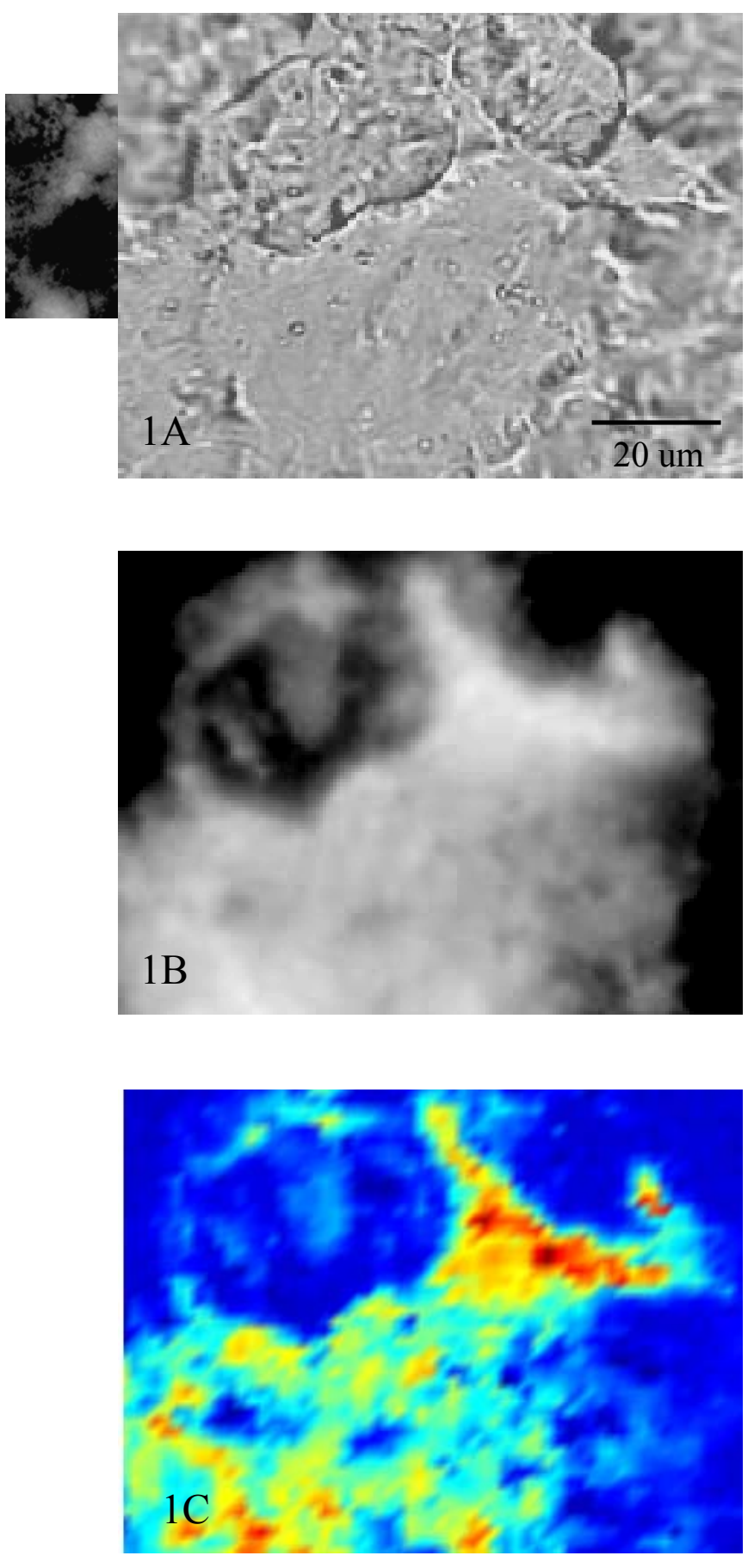

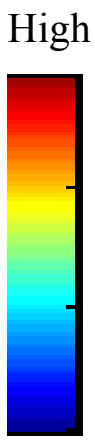

Low
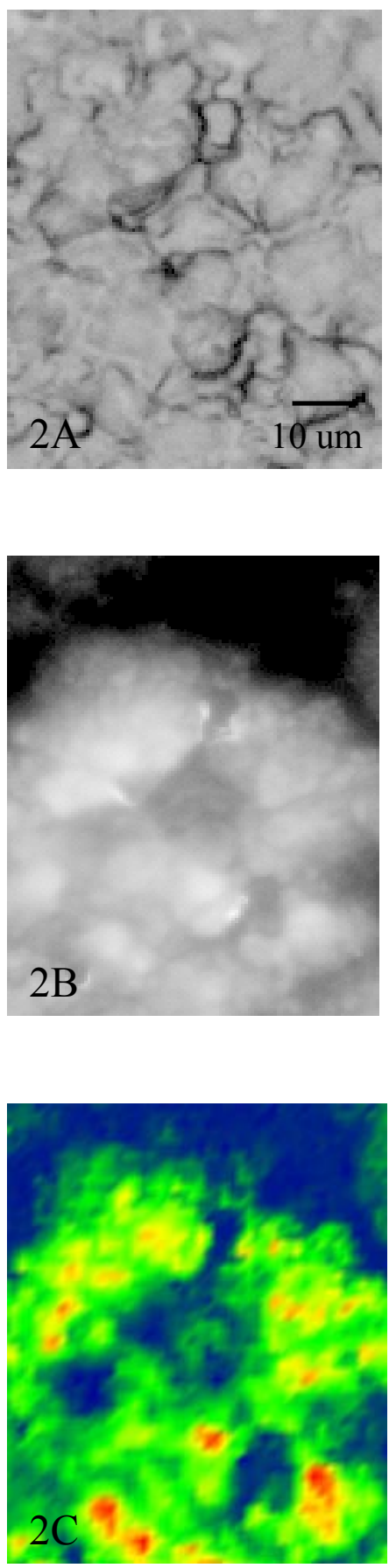

Figures 1A and 2A. Brightfield light photomicrographs of a region of a solid formulation.

Figures 1B and 2B. Reflected light fluorescence photomicrographs of the same region as 1A and 2A.

Figures $1 \mathrm{C}$ and 2C. Raman microscopy chemical maps (of the same region as in 1B and 2B) of a specific formulation component. The different colors correspond to the relative amount of the component at different locations as indicated by the color scale bar. The location map agrees with the fluorescence tagging result seen in $1 \mathrm{~B}$ and $2 \mathrm{~B}$. 